\title{
Analysis of Thin Phase-Shifter Films using Surface Analysis Techniques
}

\author{
Vincent S. Smentkowski ${ }^{1}$, Laurie LeTarte ${ }^{1}$, Hong Piao ${ }^{1}$, Michael Marko ${ }^{2}$ \\ ${ }^{1}$ General Electric Global Research Center, 1 Research Circle, Niskayuna NY 12309 \\ ${ }^{2}$ Wadsworth Center, Empire State Plaza, P.O. Box 509, Albany NY 12201-0509
}

Many types of phase-shifters have been developed for use in place of the TEM objective aperture [1]. The phase shifters act to increase phase contrast by providing high transfer of information over a very wide spatial-frequency range. Unfortunately, many of these devices fail shortly after being installed into the instrument due to charging in the electron beam, so we have been experimenting with surface deposition of novel thin-film metals. In some cases, it is essential that the electronscattering cross-section of the metal film be as small as possible, so the films must often be quite thin (less than $10 \mathrm{~nm}$ thick). Accurate analysis of such thin films is required to understand the composition of the layers, unexpected impurities both in the films and at the interfaces, the oxidation state of the layers, and the lateral uniformity of the layers.

In this paper/poster we use a suite of surface analysis techniques to monitor the distribution of species through one multilayer phase plate stack using depth profiling protocol. The pros and cons of each of the surface analysis techniques are illustrated via the sample analyzed.

The multilayer film stack under investigation here was deposited using a dual argon-ion-beam coater (South Bay ISB/e), with a built-in a crystal-thickness monitor. The composition of the thin film stack was $2 \mathrm{~nm} \mathrm{Rh}, 6 \mathrm{~nm}$ of $\mathrm{C}$, and $2 \mathrm{~nm} \mathrm{Al} \mathrm{which} \mathrm{were} \mathrm{simultaneously} \mathrm{deposited} \mathrm{on} \mathrm{both} \mathrm{Si}$ and glass substrates. The outer surface revealed a few atomic percent of each $\mathrm{F}, \mathrm{K}, \mathrm{N}$ and $\mathrm{S}$ in addition to $\mathrm{Rh}, \mathrm{C}$ and $\mathrm{O}$. An objective of this analysis was to determine if oxygen is present in the Al layer.

Figure 1 shows an AES depth profile measurement performed over an area of approximately $300 \mathrm{~nm}^{2}$. Each of the three layers are visible, the AES data reveals oxygen (and trace N) in the Al layer. ToF-SIMS reveals some inter layer mixing of the three layers. The ToF-SIMS depth profile traces shown in Figure 2 reveal the distribution of trace $\mathrm{Na}, \mathrm{Mg}, \mathrm{K}$ and $\mathrm{Ca}$ through the layer stack when a $40,000 \mathrm{~mm}^{2}$ area was analyzed.

The $\mathrm{X}$ axis for the depth profile traces shown in Figures 1 and 2 is in units of erosion time. As we know the layer thicknesses from the crystal thickness monitor, we can determine the erosion rates for the erosion conditions which include beam energy, current density angle of incidence, raster area, and the material being eroded.

Each of the three techniques used here were able to successfully identify and resolve each of the 3 layers with a sufficient number of data points defining the thin layers. Each of the techniques also revealed oxygen in the aluminum layer with a stoichiometry of about $\mathrm{Al}_{2} \mathrm{O}_{3}$.

An advantage of ToF-SIMS for depth profiling is the ability to rapidly collect an image at every depth, allowing for 3D rendering of the data sets. The 3D ToF-SIMS renderings do not reveal discontinuous regions at a length scale of a few hundred nm (the conditions used here); ruling out holes at a length scale of less than a few hundred nanometers in the Rh layer would require ToF- 
SIMS analysis using a pulsing mode with a smaller analytical spot size. Another advantage of ToFSIMS is that a full mass spectrum is saved at every voxel and hence all elements and high mass molecular fragments are analyzed. Often unexpected species are revealed sub surface. A disadvantage of ToF-SIMS is the ion yield varies depending on the composition of the matrix and this makes quantitative analysis more difficult. In contrast sensitivity factors have been developed for both AES and XPS and these reported sensitivity factors are typically valid to within $10 \%$ and hence quantitative analysis is easier.

Mike Marco acknowledges funding through NIH grant GM103555.

\section{References:}

[1] R. Glaeser, Rev. Sci. Instr. 84(2013), p. 111101.

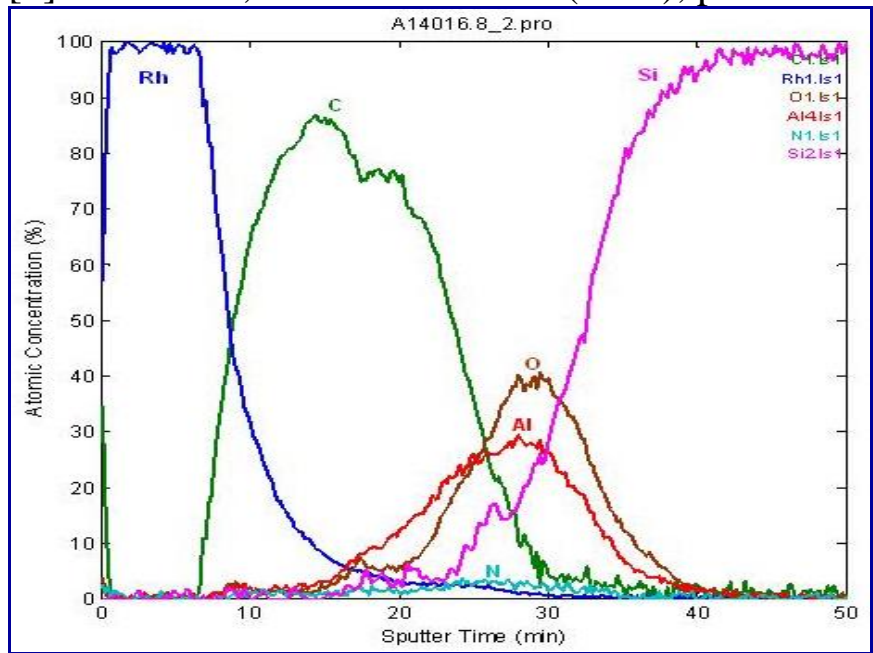

Figure 1. The depth distribution of $\mathrm{Rh}, \mathrm{C}, \mathrm{Al}, \mathrm{O}$, and $\mathrm{N}$ measured by AES. The $\mathrm{Y}$ axis is in units of atomic percent, see text for discussion.

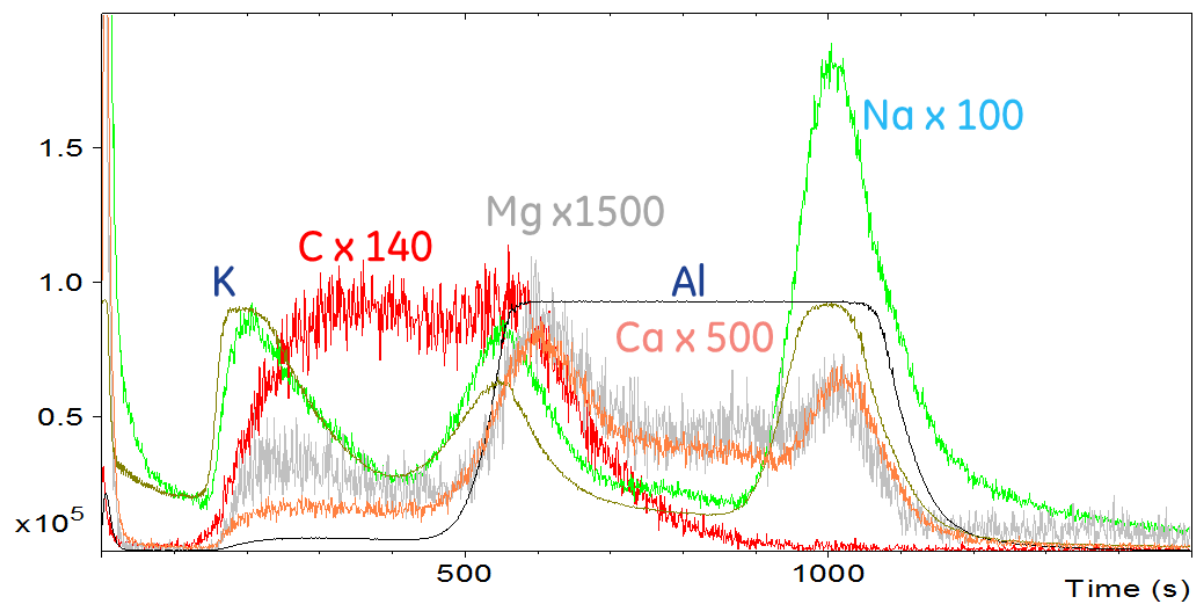

Figure 2. The depth distribution of trace species measures by ToF-SIMS. C and Al are included to define the layers. The $\mathrm{Y}$ axis is in units of peak area. Conversion to concentration would require the use of matrix matched relative sensitivity factors for each layer. 